\section{Probing a cell-embedded megadalton protein complex by DNP-supported solid-state NMR}

\author{
Mohammed Kaplan ${ }^{1}$, Abhishek Cukkemane ${ }^{1,4}$, \\ Gydo C P van Zundert ${ }^{1}$, Siddarth Narasimhan ${ }^{1}$, \\ Mark Daniëls ${ }^{1}$, Deni Mance ${ }^{1}$, Gabriel Waksman ${ }^{2}$, \\ Alexandre M J J Bonvin ${ }^{1}$, Rémi Fronzes ${ }^{3}$, \\ Gert E Folkers $^{1}$ \& Marc Baldus ${ }^{1}$
}

\begin{abstract}
Studying biomolecules at atomic resolution in their native environment is the ultimate aim of structural biology. We investigated the bacterial type IV secretion system core complex (T4SScc) by cellular dynamic nuclear polarizationbased solid-state nuclear magnetic resonance spectroscopy to validate a structural model previously generated by combining in vitro and in silico data. Our results indicate that T4SScc is well folded in the cellular setting, revealing protein regions that had been elusive when studied in vitro.
\end{abstract}

Super-high-resolution light microscopy ${ }^{1}$ and electron tomography ${ }^{2}$ have provided unprecedented insights into cellular organization at the nanometer scale. Obtaining information at the atomic level, however, typically requires modeling using in vitro data provided by X-ray crystallography, nuclear magnetic resonance (NMR) or electron microscopy. In-cell solution-state NMR can track structure provided that molecular units tumble rapidly ${ }^{3,4}$. Solid-state NMR spectroscopy (ssNMR) has been used to study small proteins embedded in native membranes ${ }^{5-8}$; we have previously studied ${ }^{9,10}$ a 150 -amino-acid membrane protein using cellular preparations. However, cellular ssNMR experiments on larger molecules or complexes pose additional challenges ranging from sample preparation to data interpretation. Here, we show that the combination of dedicated labeling schemes, cellular ssNMR and dynamic nuclear polarization (DNP) ${ }^{11}$ allows us to examine the validity of the structural model of a membrane-associated complex directly in a cellular setting (Supplementary Fig. 1a).

We studied the type IV secretion system core complex (T4SScc), a $1-\mathrm{MDa}$ protein machine consisting of 14 copies of 3 proteins
(VirB7, VirB9 and VirB10, Supplementary Fig. 1b) ${ }^{12}$. It is part of a larger machine (T4SS) that spans the periplasm and is embedded in both the inner and outer membranes of Gram-negative bacteria. The complex transports various substrates, including plasmid DNA, during bacterial conjugation or carries effector proteins into eukaryotic cells ${ }^{13}$. Structural information about T4SScc has been gathered on purified complexes using electron microscopy ${ }^{12,14}$, and atomic structures have been obtained ${ }^{15}$ for the outer layer (OL) composed of VirB7 and the C-terminal regions of VirB9 and VirB10 (Supplementary Fig. 1b). Recent work suggests that the core complex also largely maintains its structure in T4SS constructs comprising T4SScc components and the inner membrane components VirB3, VirB4, VirB5, VirB6 and VirB8, connected to the inner membrane complex via the inner layer (IL) of T4SScc and a flexible region called the stalk ${ }^{16}$. From cysteine scanning studies $^{17}$ it became evident that the IL of T4SScc contains $\mathrm{N}$-terminal protein segments of VirB10 that insert into the inner membrane, which is located at a unique position to regulate substrate transfer across the cell envelope. However, atomic information on the IL has remained elusive. In fact, a comparison of the available electron microscopy data suggests that the $\mathrm{N}$-terminal region of VirB10 is compact or unstructured in purified variants of the T4SScc and must adopt a more extended conformation reaching into the inner membrane in larger T4SS complexes and/or when embedded in the cellular envelope ${ }^{16}$.

To obtain structural information about T4SScc in its cellular setting, we coexpressed all three subunits in wild-type Escherichia coli BL21 (DE3) (WT, Supplementary Fig. 2a) as well as in cells deficient in OmpA and OmpF (ref. 9) (BL21 double mutant (dm), Supplementary Fig. 2a) following earlier procedures ${ }^{12}$. In both cases, cell envelope fractions contained T4SScc components, and electron microscopy studies after further purification revealed intact protein complexes (Supplementary Fig. 2b). Furthermore, previous work ${ }^{15}$ as well as our experimental data presented below strongly suggested that the complex is correctly folded in the cellular envelope. For cellular ssNMR studies, we prepared T4SScc either uniformly labeled with ${ }^{13} \mathrm{C}$ and ${ }^{15} \mathrm{~N}$ or selectively labeled with $\left[{ }^{13} \mathrm{C},{ }^{15} \mathrm{~N}\right]($ Gly,Ser,Leu, Val $)$ or with $\left(\left[{ }^{13} \mathrm{C},{ }^{15} \mathrm{~N}\right] \mathrm{Thr},\left[{ }^{15} \mathrm{~N}\right] \mathrm{Val}\right)$ in the cell envelope of BL21dm cells (referred henceforth to as U-, GSLV- and TV-labeled T4SScc, respectively). Specific amino acid labeling was confirmed by two-dimensional (2D) ssNMR (Supplementary Fig. 3). For spectral evaluation, we used a hybrid approach (Supplementary Fig. 1a) with a T4SScc structural model combining previous electron microscopy results and X-ray data available for the $\mathrm{OL}^{14,15}$. In particular, the electron microscopy data provided global information about the OL and its putative

\footnotetext{
${ }^{1}$ NMR Spectroscopy, Utrecht University, Utrecht, the Netherlands. ${ }^{2}$ Institute of Structural and Molecular Biology, University College London and Birkbeck, London, UK. ${ }^{3}$ Groupe à 5 ans, Biologie structurale de la secretion bacterienne, Unité mixte de recherche Centre National de la Recherche Scientifique-Institut Pasteur 3528, Institut Pasteur, Paris, France. ${ }^{4}$ Current address: Microbiology Department, Tuljaram Chaturchand College, Baramati, India. Correspondence should be addressed to G.E.F. (gert@nmr.chem.uu.nl) or M.B. (m.baldus@uu.nl).
} 
$\begin{array}{lll}\text { a } & \text { B7 } & 30 \frac{\mathrm{TV}}{31} \\ & \text { B9 } & 127 \mathrm{TV} \cdots \underline{T V}_{249}\end{array}$

B10 $\quad 57^{\mathrm{TV}} \cdots \underline{\mathrm{TV}} \ldots \mathrm{TTV}_{221}$

C
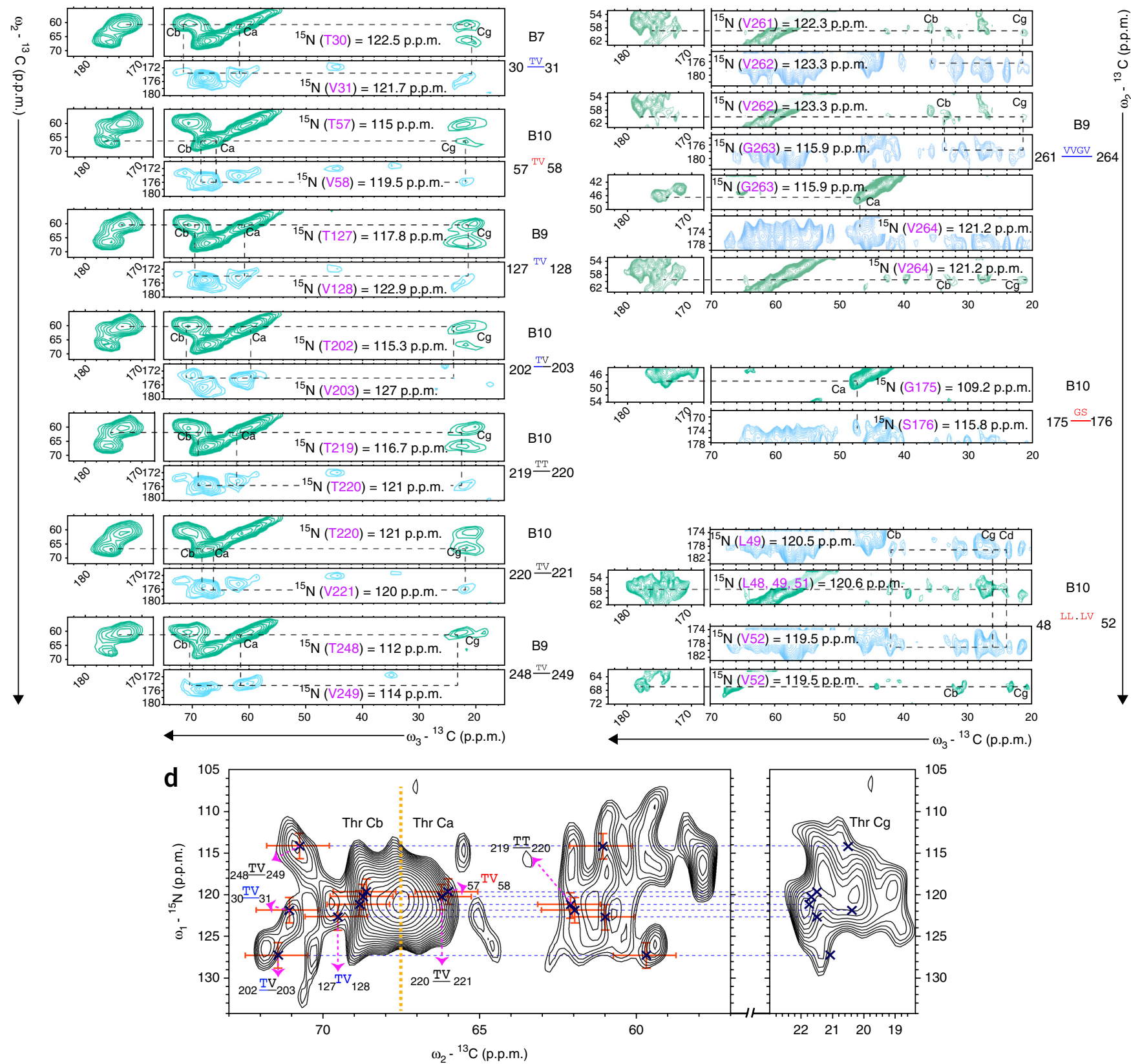

Figure 1 | Analysis of DNP-based two and three-dimensional ssNMR data sets of tailored-labeled cell-embedded T4SScc. (a,b) Predicted sequential correlations in TV-T4SScc and GSLV-T4SScc, respectively, with letters indicating correlations in random coil (black), $\beta$-strand (blue) and $\alpha$-helix (red). Underlined segments reflect crystallized T4SScc subdomains. Single dots represent 1-4 residues, double dots 5-8 residues and triple dots more than 8 residues. (c) Two-dimensional $\left({ }^{13} \mathrm{C},{ }^{13} \mathrm{C}\right.$ ) planes taken out of 3D NCACX (green) and 3D NCOCX (light blue) data sets of TV-T4SSCC acquired at 400 MHz DNP. All seven expected correlations were identified in the spectra. (d) 2D NCOCX of TV-T4SScc recorded at 800 MHz DNP conditions. Crosses represent the seven correlations expected in this labeling scheme. Additional correlations can be explained by scrambling to glycine. Orange error bars for carbon are 2 p.p.m. and for nitrogen 3 p.p.m., respectively. For clarity, the dashed vertical line separates Cb (resonating above 67.5 p.p.m.) and $\mathrm{Ca}$ (resonating below 67.5 p.p.m.) ${ }^{13} \mathrm{C}$ resonance frequencies. Data shown in e were recorded under conditions similar to those in $\mathbf{c}$ on GSLV-labeled T4SScc (see Supplementary Tables 1 and 2). In all spectra shown in c-e, dashed lines highlight carbon backbone as well as side chain correlations. 
Figure 2 | Summary of residue-specific ssNMR probes and their location in reference to the cellular envelope including the T4SScc electron microscopy map with the outer membrane complex fitted inside (PDB 3JQ0). The $\mathrm{N}$ terminus of VirB9 is also docked into the map. Identified residues in our spectra are shown as orange balls for the TV-T4SScc and red balls for the GSLV-T4SScc. Boxes represent zoom-ins of the identified correlations. Note that in the case of the $\mathrm{N}$-terminal helix of VirB10, residues given in bold were tentatively assigned. See ref. 16 for further information on the cellular location of the T4SScc subcomponents.

location relative to the outer membrane. In addition, the X-ray data were used to predict the secondary structure elements for the OL. Finally, we employed ${ }^{14}$ structure prediction servers (Supplementary Figs. 1b and 4) for T4SScc regions for which secondary structural information was lacking. Following earlier work ${ }^{9,10}$, an SDS-PAGE analysis (Supplementary Fig. 5) and a comparison of ssNMR data of cell envelopes without and with protein expression (Supplementary Fig. 6) confirmed that T4SScc and Braun's lipoprotein (Lpp) are dominant. Subsequently, we adapted the NMR software package FANDAS ${ }^{18}$ to predict the spectra of T4SScc, Lpp and the other dominating cellular envelope compounds ${ }^{9}-$ lipopolysaccharides, peptidoglycans and lipids (phosphatidylethanolamine) - that all contribute to the ssNMR spectrum of uniformly ${ }^{13} \mathrm{C},{ }^{15} \mathrm{~N}$-labeled cellular envelope preparations (Supplementary Fig. 7).

We exploited the well-established correlation between NMR resonance frequencies and protein secondary structure (see, for example, ref. 19) to obtain structural information about the backbone fold of cell-embedded T4SScc. Our optimized selective amino acid labeling was designed to maximize spectral dispersion under low-temperature DNP conditions and, at the same time, generate unambiguous structural reporters throughout VirB7, VirB9 and VirB10. For example, TV-T4SScc should give rise to seven sequential correlations in $\beta$-strand (blue), $\alpha$-helical (red) and random-coil (rc) regions (black) of the entire 1-MDa complex (Fig. 1a). Five of these correlations stem from the previously crystallized OL segments (Fig. 1a), and two sequential correlations originate from the structurally elusive regions in VirB9 and VirB10. For GSLV-T4SScc, we expected 55 sequential correlations, with 31 from the OL (Fig. 1b) and 20 originating from N-terminal VirB9 and VirB10 (Fig. 1b). Lpp sequential correlations do not contribute to our spectral analysis presented in Figure 1 (Supplementary Fig. 8).

We prepared ssNMR samples amenable to DNP studies at 400 and $800 \mathrm{MHz}$ using AMUPol${ }^{20}$ (Supplementary Fig. 9). We observed a DNP enhancement factor of $\sim 60$, enabling 3D intraresidue (NCACX) and inter-residue (NCOCX) experiments at $400 \mathrm{MHz}$ (Fig. 1c; see Online Methods and Supplementary Fig. 9). At $800 \mathrm{MHz}$, the DNP enhancement factor of $\sim 15$ allowed for 2D NCACX and NCOCX (Fig. 1d) experiments for crossvalidation. For TV-T4SScc, a combined evaluation of these data sets confirmed the presence of resonances of $\alpha, \beta$ and $\mathrm{rc}$ conformations (Fig. 1c,d and Supplementary Fig. 10). Using FANDAS and empirically determined standard deviations ${ }^{21}$ for protein secondary

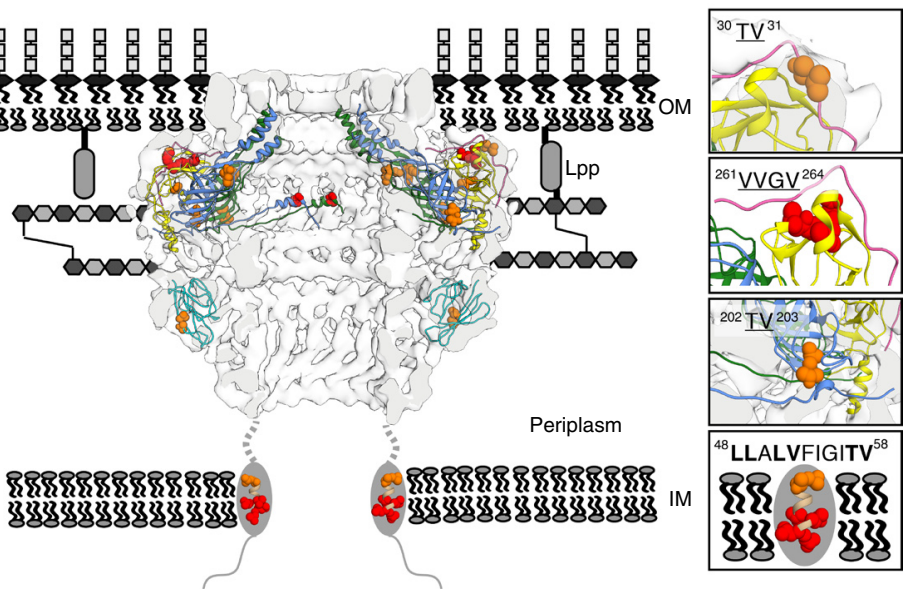

structure predictions, we identified sequential strips connected via a unique ${ }^{15} \mathrm{~N}$ chemical shift along $\omega_{1}$ in $\left(\omega_{2}, \omega_{3}\right)$ intraresidue $2 \mathrm{D} \mathrm{CA}-\mathrm{CX}$ and $\mathrm{CO}-\mathrm{CX}$ planes in our $3 \mathrm{D}$ data sets (Fig. 1c), respectively. The chemical shifts were also consistent with a 2D NCOCX experiment at $800 \mathrm{MHz}$ (Fig. 1d) and led to tentative assignments for all sequential pairs (Supplementary Table 1). The increased spectral resolution (see, for example, Supplementary Fig. 11 for a comparison of $400-$ and $800-\mathrm{MHz}$ DNP spectra) revealed several resolved correlations in the $C \beta$ region and allowed us to assess the spectral line width. For correlations such as ${ }^{202} \mathrm{ThrVal}^{203}$ (B10) in the OL or ${ }^{30} \mathrm{ThrVal}^{31}$ located in $\mathrm{B} 7$, we determined ${ }^{13} \mathrm{C}$ and ${ }^{15} \mathrm{~N}$ resonance line widths of $\sim 1.5$ p.p.m. and 3 p.p.m., respectively. Two correlations, relating to $\alpha$-helical elements predicted for ${ }^{57} \mathrm{ThrVal}^{58}$ and ${ }^{220} \mathrm{ThrVal}^{221}$ in VirB10, exhibited larger line width, especially in the ${ }^{15} \mathrm{~N}$ dimension, consistent with variable dynamics within the T4SScc.

Next, we investigated the validity of our structural model by comparing structural predictions to 3D data on GSLV-T4SScc (Fig. 1e) as in Figure 1c. Again we could identify $\omega_{2}-\omega_{3}$ planes leading to sequential correlations in the ${ }^{261}$ ValValGlyVal ${ }^{264}$ VirB9 stretch of the OL forming a $\beta$-strand in crystals, in agreement with the observed chemical shifts. NMR data and predictions were also consistent with the detection of the only glycineserine pair occurring in an $\alpha$-helix in $\left({ }^{175} \mathrm{GlySer}^{176}\right)$ in VirB10. We expected the sequence $\left({ }^{40}\right.$ KAFVILMALLALVFIGITV $\left.{ }^{58}\right)$ in the $\mathrm{N}$-terminal region of VirB10 to adopt an $\alpha$-helical conformation (Fig. 1b). Indeed, we could identify in our 3D data correlations consistent with leucine-leucine (only found in the N-terminal B10) or leucine-valine contacts (Fig. 1e and Supplementary Table 2), in line with the identification of the unique $\alpha$-helical ${ }^{57} \mathrm{ThrVal}^{58}$ pair for N-terminal VirB10 (Fig. 1c,d). ssNMR experiments at higher temperatures provided evidence against a sizable increase in protein motion for the N-terminal half of VirB10 (Supplementary Fig. 12) and thus suggested that this N-terminal VirB10 stretch is membrane associated.

Taken together, our results strongly suggest that the 3D fold of the T4SScc OL seen in in vitro crystals is maintained in the cellular preparation (Fig. 2). At the same time, these findings underlined the validity of our chemical-shift analysis as a means to probe protein structure in a cellular environment. Moreover, our observed correlations in the IL part of T4SScc were consistent with a $\beta$-strand arrangement for ${ }^{127} \mathrm{ThrVal}^{128}$ in VirB9, providing the first experimental atomic evidence that the $\mathrm{N}$-terminal 
region of VirB9 adopts a distinct conformation at the periplasmic surface of the cell-embedded core complex. Also, we could identify a series of sequential correlations ( ${ }^{48}$ LLALVFIGITV $^{58}$ ) of the IL suggesting that VirB10 inserts into the inner membrane as an $\alpha$-helix, allowing the core complex to span the entire cell envelope (Fig. 2). Our results also indicate that VirB9 and VirB10 are involved in forming a stable scaffold that allows the assembly of the entire complex in the cellular envelope. At the same time, differences in ssNMR line width seen in our study suggest structural flexibility that may be needed for accommodation of the pilus and substrate secretion ${ }^{22}$.

Existing approaches rely on the molecular modeling of in vitro data into low-resolution cellular structures. The combination of such approaches with cellular ssNMR data such as shown here allows for direct experimental validation of atomic models in a cellular environment. In the case of T4SScc, such information may help to engineer systems as in vivo gene-delivery vectors, with applications in DNA vaccination and therapeutic gene therapy in human cells ${ }^{23}$. Furthermore, our approach may be applicable to studying molecular complexes at the atomic level in their native environment, including those occurring during gene transcription, protein folding or cellular signaling.

\section{METHODS}

Methods and any associated references are available in the online version of the paper.

Accession codes. Protein Data Bank: PDB 3JQO, 1EQ7 and 2YPW.

Note: Any Supplementary Information and Source Data files are available in the online version of the paper.

\section{ACKNOWLEDGMENTS}

We thank E. Koers and J. van der Zwan for helpful discussions and technical support. We are indebted to P. Tordo and his group for providing AMUPol. This work was funded in part by the Netherlands Organization for Scientific Research (NW0, grants 700.26 .121 and 700.10 .443 to M.B.)

\section{AUTHOR CONTRIBUTIONS}

G.E.F. and M.B. designed the research. M.K., M.D., A.C. and S.N. produced samples, and M.K. and D.M. performed ssNMR experiments. M.K., G.C.P.v.Z. and A.M.J.J.B. docked atomic models into the electron microscopy density map. R.F. provided clones and conducted electron microscopy studies. G.W. and M.B. wrote the paper and all authors edited it.

\section{COMPETING FINANCIAL INTERESTS}

The authors declare no competing financial interests.

Reprints and permissions information is available online at http://www.nature. com/reprints/index.html.

1. Huang, B., Babcock, H. \& Zhuang, X. Cell 143, 1047-1058 (2010).

2. Leis, A., Rockel, B., Andrees, L. \& Baumeister, W. Trends Biochem. Sci. 34, 60-70 (2009).

3. Serber, Z., Corsini, L., Durst, F. \& Dötsch, V. Methods Enzymol. 394, 17-41 (2005).

4. Banci, L., Barbieri, L., Luchinat, E. \& Secci, E. Chem. Biol. 20, 747-752 (2013).

5. Etzkorn, M. et al. Angew. Chem. Int. Edn Engl. 46, 459-462 (2007).

6. Fu, R. et al. J. Am. Chem. Soc. 133, 12370-12373 (2011).

7. Jacso, T. et al. Angew. Chem. 124, 447-450 (2012).

8. Miao, Y. et al. Angew. Chem. Int. Edn Engl. 51, $8383-8386$ (2012).

9. Renault, M. et al. Proc. Natl. Acad. Sci. USA 109, $4863-4868$ (2012).

10. Renault, M. et al. Angew. Chem. Int. Edn Engl. 51, 2998-3001 (2012).

11. Ni, Q.Z. et al. Acc. Chem. Res. 46, 1933-1941 (2013).

12. Fronzes, R. et al. Science 323, 266-268 (2009).

13. Low, H.H. et al. Nature 508, 550-553 (2014).

14. Rivera-Calzada, A. et al. EMBO J. 32, 1195-1204 (2013).

15. Chandran, V. et al. Nature 462, 1011-1015 (2009).

16. Trokter, M., Felisberto-rodrigues, C., Christie, P.J. \& Waksman, G. Curr. Opin. Struct. Biol. 27, 16-23 (2014).

17. Jakubowski, S.J. et al. Mol. Microbiol. 71, 779-794 (2009).

18. Gradmann, S. et al. J. Biomol. NMR 54, 377-387 (2012).

19. Shen, Y., Delaglio, F., Cornilescu, G. \& Bax, A. J. Biomol. NMR 44 213-223 (2009).

20. Sauvée, C. et al. Angew. Chem. Int. Edn. Engl. 52, 10858-10861 (2013).

21. Seidel, K., Etzkorn, M., Schneider, R., Ader, C. \& Baldus, M. Solid State Nucl. Magn. Reson. 35, 235-242 (2009).

22. Waksman, G. \& Orlova, E.V. Curr. Opin. Microbiol. 17, 24-31 (2014).

23. Llosa, M., Schröder, G. \& Dehio, C. Trends Microbiol. 20, 355-359 (2012). 


\section{ONLINE METHODS}

Sample preparation. BL21 WT and BL21 double mutant ${ }^{9}$ cells were transformed with pKM101 following earlier work ${ }^{12}$ and plated on LB agar plates enriched with glucose $(4 \mathrm{~g} / \mathrm{L})$ and containing chloramphenicol (35 mg/L). Next day, a fresh colony of BL21 WT or BL21dm cells was inoculated in a $2 \mathrm{ml} \mathrm{LB}$ culture and grown at $37^{\circ} \mathrm{C}$ until it reached $\mathrm{OD}_{600} \sim 1$. Thereafter, the cells were centrifuged and transferred into $25 \mathrm{ml}$ of unlabeled $\mathrm{M} 9$ medium. At $\mathrm{OD}_{600} \sim 1$, cells were centrifuged again and transferred into $250 \mathrm{ml}$ of unlabeled M9 medium. Upon reaching $\mathrm{OD}_{600} \sim 1$, the cells were centrifuged for $10 \mathrm{~min}$ at $2,000 \mathrm{~g}$ and transferred into $250 \mathrm{ml}$ of ${ }^{13} \mathrm{C},{ }^{15} \mathrm{~N}$-labeled M9 medium in the case of uniformly labeled samples and then induced with tetracycline $(200 \mu \mathrm{g} / \mathrm{L}$, see ref. 12). After induction, samples were incubated at $20^{\circ} \mathrm{C}$ over night. 5-6 h after induction, another $200 \mu \mathrm{g} / \mathrm{L}$ of tetracycline and $35 \mathrm{mg} / \mathrm{L}$ chloramphenicol were added. In case of GSLV-T4SScc samples, $200 \mathrm{mg} / \mathrm{L}$ of each labeled amino acid was added to unlabeled M9 medium at $\mathrm{OD}_{600} \sim 1,20$ min after induction with tetracycline. In TV-T4SScc sample, in addition to the labeled amino acids, all other amino acids were added (as unlabeled at $200 \mathrm{mg} / \mathrm{L}$ ) to the medium after induction to decrease scrambling. Cell lysis and cell envelope samples were prepared as described earlier ${ }^{9}$. The T4SScc was further purified as in ref. 12 and visualized on a Tecnai T12 $120 \mathrm{kV}$ BioTwin electron microscope after negative staining with $2 \%$ uranyl acetate.

For DNP measurements, the cell envelope samples were washed twice with a buffer containing $20 \mathrm{mM}$ AMUPol in $32.5 \% \mathrm{D}_{2} \mathrm{O}$, $12.5 \% \mathrm{H}_{2} \mathrm{O}, 10 \% 100 \mathrm{mM}$ HEPES $(\mathrm{pH}=7)$ and $45 \%$ glycerol d8 for GSLV-T4SScc and 22.5\% glycerol d8 for TV-T4SScc. $50 \mu \mathrm{l}$ of this buffer was used in each washing step. Samples were centrifuged after washing $(100,000 \mathrm{~g}, 25 \mathrm{~min})$.

Solid-state NMR \& DNP experiments. For NMR measurements, a standard-bore $700 \mathrm{MHz}$ as well as wide-bore $800 \mathrm{MHz} / 527 \mathrm{GHz}$ DNP and $400 \mathrm{MHz} / 263 \mathrm{GHz}$ DNP systems (Bruker Biospin) were used. We filled cellular envelope preparations containing approximately $1 \mathrm{mg}$ of ${ }^{13} \mathrm{C},{ }^{15} \mathrm{~N}$-labeled T4SScc into standard $3.2 \mathrm{~mm}$ rotors. For all DNP measurements, samples were cooled down to $100 \mathrm{~K}$ in a $3.2 \mathrm{~mm}$ sapphire rotor. The DNP enhancement was measured by overlaying $\mathrm{HC} \mathrm{CP} / \mathrm{MAS}$ spectra recorded with and without microwave irradiation. Two- and three-dimensional NC correlation spectra were recorded using SPECIFIC-CP ${ }^{24}{ }^{15} \mathrm{~N}-{ }^{13} \mathrm{C}$ transfers. Homonuclear $\left({ }^{13} \mathrm{C},{ }^{13} \mathrm{C}\right)$ transfers were established using PARIS ${ }^{25}$ or spin-diffusion ${ }^{26}$ blocks. ${ }^{1} \mathrm{H}$ decoupling using SPINAL64 (ref. 27) was employed during evolution and detection periods.

Additional experimental parameters. Note that in all experiments, heteronuclear SPECIFIC transfer ${ }^{24}$ was established between $t_{1}$ and $t_{2}$ and homonuclear $\left({ }^{13} \mathrm{C},{ }^{13} \mathrm{C}\right)$ mixing was established between $t_{2}$ and $t_{3}$. ${ }^{1} \mathrm{H}$ decoupling using SPINAL64 was employed at $87 \mathrm{kHz}$ during evolution and detection periods.

Additional experimental parameters are given below.

The NCACX in Figure 1c (400 MHz DNP) was acquired using 14 points in $t_{2}$ and 10 points in $t_{1}$ with a spectral width of $5,027 \mathrm{~Hz}$ and $2,269 \mathrm{~Hz}$ in $t_{2}$ and $t_{1}$ respectively. The spectrum was processed using a squared sine function 3 in $t_{3}$ and $t_{2}$ and sine bell 2 in $t_{1}$, with zero filling 256 in $t_{3}$ and 64 in $t_{2}$ and $t_{1}$, with 4 linear prediction coefficients in $t_{1}$ and $t_{2}$.
The NCOCX in Figure 1c (400 MHz DNP) was acquired using 10 points in $t_{1}$ and $t_{2}$ with spectral width of $5,027 \mathrm{~Hz}$ and $2,269 \mathrm{~Hz}$ in $t_{2}$ and $t_{1}$ respectively. The spectrum was processed using a squared sine function 3 in $t_{3}$ and $t_{2}$ and sine bell 2 in $t_{1}$, with zero filling 256 in $t_{3}$ and 64 in $t_{2}$ and $t_{1}$, with 4 linear prediction coefficients in $t_{1}$ and $t_{2}$.

The NCOCX in Figure 1d ( $800 \mathrm{MHz}$ DNP) was acquired using $16 t_{1}$ points with a spectral width of $4,055 \mathrm{~Hz}$ in $t_{1}$. The spectrum was processed using squared sine 3.5 function in both $t_{1}$ and $t_{2}$ with $2 \mathrm{k}$ and $1 \mathrm{k}$ zero filling points in $t_{2}$ and $t_{1}$ respectively.

The NCACX in Figure 1e (400 MHz DNP) was acquired using 14 points in $t_{1}$ and $t_{2}$ with spectral width of $5,027 \mathrm{~Hz}$ and $2,269 \mathrm{~Hz}$ in $t_{2}$ and $t_{1}$ respectively. The spectrum was processed using a squared sine function 3 in $t_{3}$ and $t_{2}$ and a sine bell function 2 in $t_{1}$, with zero filling 256 in $t_{3}$ and 64 in $t_{2}$ and $t_{1}$, with 8 linear prediction coefficients in $t_{1}$ and $t_{2}$.

The NCOCX in Figure 1e ( $400 \mathrm{MHz}$ DNP) was acquired using 15 points in $t_{1}$ and $t_{2}$ with spectral width of $5,027 \mathrm{~Hz}$ and 2,269 $\mathrm{Hz}$ in $t_{2}$ and $t_{1}$ respectively. The spectrum was processed using a sine bell function 3 in $t_{3}$ with $2 \mathrm{k}$ zero filling. In $t_{2}$ and $t_{3}$ the spectrum was processed with sine bell function 2 and 64 zero filling in both dimensions, with 4 linear prediction coefficients. Further experimental parameters relating to Figure 1 and Supplementary Figures 3, 6, 7 and 11 are given in Supplementary Tables 3-8.

Modeling and structural analysis. NMR signals were predicted using crystal structures of the outer membrane complex crystal structure (PDB 3JQO) and modeled inner membrane complex. The secondary structure of the $\mathrm{N}$ termini of B9 and B10 were predicted using Jpred3 (ref. 28) and PSIPRED ${ }^{29}$. Signals stemming from Braun's lipoprotein (Lpp) were predicted using the Lpp crystal structure (PDB $1 \mathrm{EQ}^{30}$ ). Chemical shift predictions for the crystal part of T4SScc was made using ShiftX ${ }^{31}$ and NMR correlations were derived by FANDAS ${ }^{18}$. Analysis of the NMR/ DNP spectra was performed using Sparky (https://www.cgl.ucsf. edu/home/sparky/).

The atomic model of the outer membrane complex of T4SScc (PDB 3JQO) was fitted into the electron microscopy (EM) density (EMD-2232) ${ }^{14}$ as a rigid body using UCSF Chimera ${ }^{32}$. The predicted transmembrane helix in the N-terminal part of VirB10 was modeled by imposing a helical conformation on the amino acid sequence and then manually placed into the membrane. For the non-crystalline N-terminal part of VirB9, the already docked atomic model (PDB 2YPW) ${ }^{14}$ in the EM density $(\mathrm{EMD}=2,232)$ was used. Atomic models of the T4SS core complex in the EM density and of the N-terminal transmembrane helix of B10 are available as Supplementary Data.

\footnotetext{
24. Baldus, M., Petkova, A.T., Herzfeld, J. \& Griffin, R.G. Mol. Phys. 95, 1197-1207 (1998).

25. Weingarth, M., Demco, D.E., Bodenhausen, G. \& Tekely, P. Chem. Phys. Lett. 469, 342-348 (2009).

26. Bloembergen, N. Physica 15, 386-426 (1949).

27. Fung, B.M., Khitrin, A.K. \& Ermolaev, K. J. Magn. Reson. 142, 97-101 (2000).

28. Cole, C., Barber, J.D. \& Barton, G.J. Nucleic Acids Res. 36, W197-W201 (2008).

29. Buchan, D.W., Minneci, F., Nugent, T.C.O., Bryson, K. \& Jones, D.T. Nucleic Acids Res. 41, W349-W357 (2013).

30. Shu, W., Liu, J., Ji, H. \& Lu, M. J. Mol. Biol. 299, 1101-1112 (2000).

31. Neal, S., Nip, A.M., Zhang, H. \& Wishart, D.S. J. Biomol. NMR 26, 215-240 (2003).

32. Pettersen, E.F. et al. J. Comput. Chem. 25, 1605-1612 (2004).
} 